\title{
E-HEALTH: FROM SENSORS TO SYSTEMS
}

\author{
Giovanni De Micheli \\ EPFL, Lausanne, Switzerland
}

\begin{abstract}
Electronic-health or E-health is a broad area of engineering that leverages transducer, circuit and systems technologies for applications to health management and lifestyle. Scientific challenges relate to the acquisition of accurate medical information from various forms of sensing inside/outside the body and to the processing of this information to support or actuate medical decisions. Ehealth systems must satisfy safety, security and dependability criteria and their deployment is critical because of the low-power and low-noise requirements of components interacting with human bodies. E-health is motivated by the social and economic goals of achieving better health care at lower costs and will revolutionize medical practice in the years to come.
\end{abstract}

\section{KEYWORDS}

E-health, sensor, biosensor, drug administration, implant, decision support

\section{INTRODUCTION}

Bettering health care and lowering its costs is a pressing economic and social issue, and it is part of the agenda of the governments of most countries in the world. The rapid expansion of distributed communication and computing systems, with low-cost terminals (such as smart telephones) suggests that electrical and information technology can address many health management issues. In parallel, recent discoveries in biology and medicine have made these sciences more quantitative, and thus the gap between medical and engineering sciences has narrowed.

Electronic-health, or E-health, has raised many hopes to both bring better medical technologies to the patients as well as to rationalize and lower health costs. Still, the path to make recent discoveries into standard medical practice is long and requires efforts at various levels, from scientists to practitioners and to legislators.

Health has to be understood within a broad perspective. The World Health Organization (WHO) defines health as "a state of complex physical, mental and social well-being and not merely the absence of disease and infirmity". For this reason, E-health can address various social and market segments with different requirements, such as: i) devices and systems for monitoring and enhancing the well-being of active people, like sportspeople; ii) monitoring devices for the weak sectors of the population, such as the elderly, the handicapped and those affected by chronic but mild pathologies; iii) therapeutic means for diagnosing, monitoring and treating peoples with infirmities. Within these three sectors, barriers to adopt new technologies vary widely, as risks, costs and projected success rates change.

E-health provides the instrument to address some of the recent trends in medicine. Predictive medicine exploits the wealth of data provided by omics technologies. This field has grown because of the dropping costs of human DNA sequencing. Personalized medicine addresses cures that are tuned to the patient, and thus requires detailed data acquisition from the patient. Preventive medicine focuses on the quality of aging through appropriate nutrition and lifestyle, thus requiring also quantitative monitoring. Participative medicine relates to sharing data and experiences through means like social media, thus requiring selecting and classifying medical information. All these trends in medicine benefit and require data acquisition, processing and networking technologies.

\section{HEALTH MANAGEMENT}

Future health management systems will require an increasingly larger presence of automation, information extraction and elaboration, as well as control of the medical procedures. In essence, we can envision three major areas that require innovation: i) real-time sensing and data acquisition of bio-chemical compound concentrations; ii) information networking through a specialized physical layer; iii) data elaboration, retrieval and decision-making support and/or actuation.

Sensing is a discipline that traditionally has been developed by communities related to fundamental sciences (e.g., physics, chemistry and biology). Despite the large number of sensors available, their effective use is limited by size, power consumption and lack of effective integration with electronic and information systems. In other words, most medical data acquisition systems are still based on discrete components, much like transistor radios were assembled fifty years ago. The integration of sensing with electronics, and thus the merging of sensing and electronic design, is key to achieving miniaturized, low-power, lownoise data acquisition chains with detection limits in regions of interest for clinical studies. To date, only glucose monitoring has reached some form of maturity and some FDA-approved devices are available for diabetic patient monitoring.

The challenges of biomedical electronic systems are related to both data acquisition and communication. Indeed, sensors in the body need to communicate to external devices. Power delivery means can obviate the need of implanted batteries, which always present some risk factor. Sensors on the body communicate through Body-Area Networks (BANs), a new technology with several challenges, including energy-efficiency, bandwidth and security. Biocompatibility and the selection of materials 
and related technologies are also important topics of research.

Information systems for biomedical applications have been developed, but they are typically used off line. The need for fast responses and their safe and secure interaction with electronics on the body and/or in the body is still an area of study. Nevertheless, the combination of networked databases with on-line data acquisition chains opens the door to better therapy as well as to promoting the autonomy of the patient and convalescent.

\section{SENSING}

Sensing and bio-sensing technologies have evolved through the years, but still a large improvement is required for sensors to become effective components of E-health chains. Most sensing technologies are based on mechanical, optical and electrical technologies [12], each having advantages and disadvantages, because of different levels of maturity. Nevertheless, the dominant presence of siliconbased processing circuits and systems suggests that electrical sensors compatible with semiconductor manufacturing technologies will eventually provide the front-end of integrated data-acquisition chains. Indeed, integration of sensing and elaboration on the same platform, possibly monolithic, is the key to reduce unit costs as well as manufacturing variability. Moreover, design methodologies that standardize sensing cells on (or over) silicon and reminiscent of microelectronic circuit semicustom approaches are key to reducing non-recurring engineering (NRE) costs in the development of electronic sensors. Indeed it is possible to design sensing cells as units of a standard cell library, where each cell is parameterizable in terms of range and other attributes. Similarly, it is possible to envision arrays of sensing cells that can be programmed after manufacturing or even at the moment of use (e.g., at point of cares). The design and use of field programmable sensing arrays (FPSAs) will be the counterpart of field programmable gate arrays (FPGAs) and enable flexible and low-cost electronic sensing.

To date only few wearable/implantable systems are available on the market. Most use non-invasive technologies to detect vital signs (by means of accelerometers, heartbeat monitoring, infrared reflectivity, etc.) and are designed for the well-being and lifestyle market. Continuous (or frequent) monitoring of chronic patients, realized at a distance (e.g., telemedicine) can reduce hospitalization time and costs as well as better the patient's life. Some pathologies require monitoring molecules in human fluids and accurate measures can be achieved by invasive means only. In the case of diabetes, some telemetry systems exist that use a wearable station coupled with a needle [13].

A possible evolution of these systems is related to the miniaturization of sensing probes and related circuits, and thus to the realization of intelligent subcutaneous implants [6]. At the same time, monitoring some pathologies requires measuring simultaneously multiple substances. Thus, scientific challenges relate to designing means for monitoring accurately multiple substances with detection limits and ranges within the therapeutic window of interest.

An example and prototype of a future implantable sensing system for monitoring human metabolism [3] is shown in Figure 1. Examples of molecules that can be monitored include glucose, lactate, cholesterol, ATP, glutamate and others. The system consists of: i) an implantable integrated sensor array and data acquisition electronic unit; ii) a wearable station for remote powering and signal processing; iii) a remote station for data collection and storage. The implant is housed in a biocompatible cylinder of about $3 \mathrm{~mm}$ of diameter and $20 \mathrm{~mm}$ in length, to be placed in the interstitial tissue. The current prototype includes: a sensor array, a CMOS mixed-signal chip and a tridimensional integrated coil for receiving inductive power and transmitting data via backscattering. The sensor array is realized with an innovative technology, where Carbon NanoTube (CNT)-nanostructured electrodes enable us to measure metabolites with increased sensitivity and lower detection limits as compared to the state of the art [2]. The integrated electronic and sensor array requires 0.5 $m W$ to operate: the electronic power is harvested by the coil. An electronic patch on the body produces the inductive field to power the implant, receives the backscattered data, and transmits it to a base station using the Bluetooth standard.

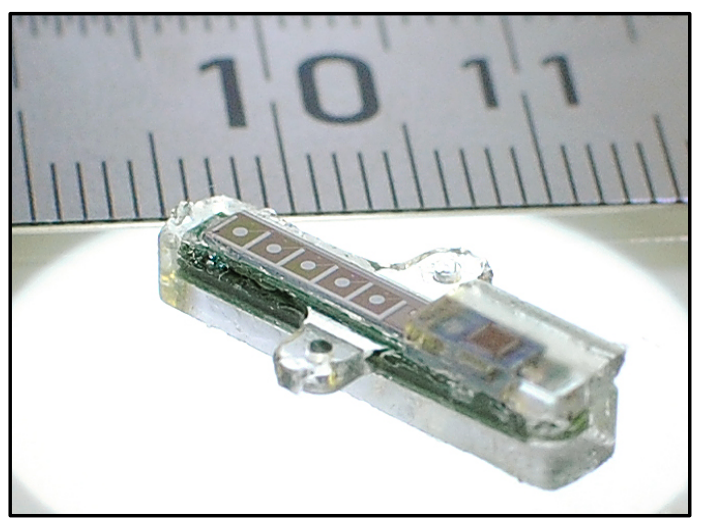

Figure 1: The I-Ironic implant as encapsulated for in-vivo tests in mice [3].

\section{DRUG ADMINISTRATION}

Clinical treatment is largely based on drug administration, mainly effected by the patient or practitioner and only partially personalized to the patient. Sensors, actuators and control systems will eventually revolutionize clinical therapy, by providing means of tightly controlling the time and dose of drug administration as well as by automating the process. Such approaches will enhance the efficacy of drugs while limiting undesirable side effects. Today most variability in drug response (roughly 80\%) resides in the pharmacokinetic (PK) phase, i.e. in doseconcentration relationships. Drug doses are typically strongly quantized (for packaging and handling reasons) and drug dosing is based upon heuristics. 


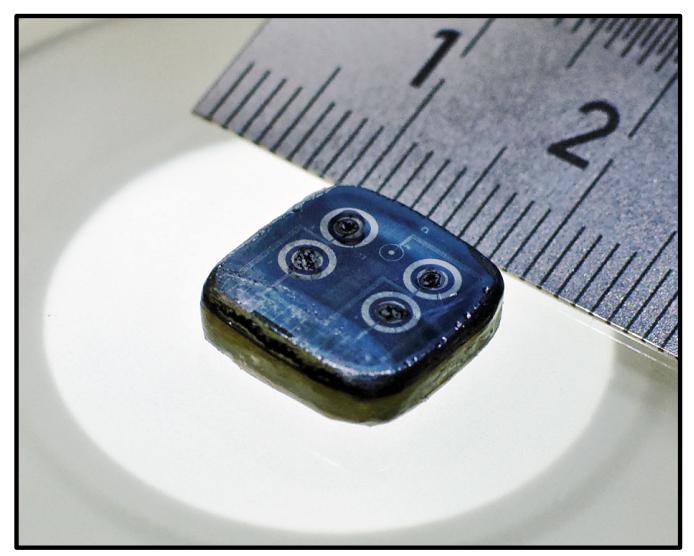

Figure 2: Implant to monitor infused drug concentration in laboratory animals [1].

Indeed, in current clinical pharmacology, the initial drug dose is chosen on the basis of previous medical experiences. It can be consequently modified based on the presence of adverse events or non-responsiveness of a patient to the treatment. However, this experience-driven method is not suitable to many kinds of drugs. There is a class of medicines, e.g., drugs for treating HIV, cancer, etc., whose effective therapeutic concentration range is quite narrow and therefore there is a very high risk to under- or over-dose a patient. Under-dosing will lead to an ineffective treatment, while over-dosing will expose the patient to a risk of toxicity. Thus controlling the drug concentration to be within the therapeutic range is essential to properly carry out the clinical monitoring; in other words, it is necessary to know how the human body affects the drug assimilation. The use of sensors, to measure drug concentration in the bloodstream [1] and of actuators (such as infusion pumps) can address the personalization and automation of drug administration but only through a specific decision and/or control system.

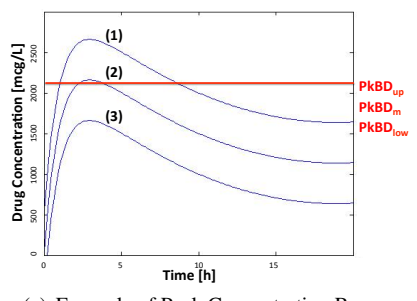

(a) Example of Peak Concentration Range

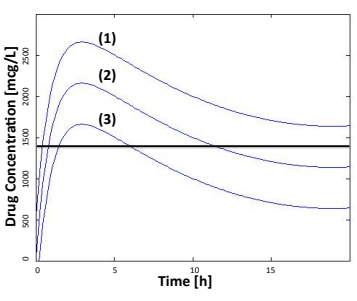

(b) Example of Trough Concentration Range
Figure 3: Examples of drug concentration curves for various doses intersecting the peak (a) and trough (b) therapeutic ranges [15].

Medical decisions are critical to patients' health and to the effective use of medical resources [4]. Several problem arise: i) the definition of a formal protocol for a given drug (e.g., Imatinib [11]) and its validation; ii) the decision of a therapy for a given patient; iii) the realization of a therapy by means of a drug administration system. There is a plethora of approaches to address these important and multifaceted problems [11]. Clinical Decision Support Systems (CDSSs) are computer-based information systems that support clinical decision-making according to the characteristics of an individual patient [8]. CDSS are based on a computerized clinical knowledge and/or expertise and can generate recommendations for specific patients [7]. In particular, a specific class of health-care procedures within CDSS address the personalized computation of a suitable drug dose for a new patient based on the prediction of the blood drug concentration by taking into account patient's features. There have been several models developed in support of PK studies that are able to predict the drug concentration in the blood. These models can be classified as analytical, statistical and based on machine learning. Analytical models are able to account only for the variables with real values, while binary-valued variables, such as gender, create strong discontinuities in the models and are in general not taken into account by the methods. Statistical approaches, including Bayesian approach [14], require the knowledge of the data distributions, such as mean and/or deviation values. For newly-developed drugs which do not have a sufficient study on the patients, it is difficult to assess a proper mean or deviation value to compute the concentrations for the patients. Systems based on machine learning, e.g., Support Vector Machine (SVM) based algorithms [15], can be very effective in predicting drug concentration in presence of parameters of various types. Within this framework, an advanced Drug Administration Decision Support System (DADSS) can assist medical doctors in decision-making regarding the drug dose adaptation during different phases of the treatment [11]. DADSS is able to recommend the dose and the intake time interval for a new patient in a personalized manner.

\section{SYSTEM-LEVEL ISSUES}

The design of health management systems and their operation must satisfy requirements typical of life-critical systems. In particular, such systems must be safe, secure and dependable.

Safety means that the system must present no treat to patients and to operators. Safety relates to the use of specific bio-compatible materials as well as containing possibly harmful substances. The operation of the overall system must be safe for the patient. In the specific case of DADSS, drug dispensing must fit the safety margins under all environmental conditions. Thus two conditions must be satisfied. The therapeutic protocol must be safe, which can be guaranteed only in the case that a formal model [5] is available and that it is formally verified. Then the implementation of the DADSS must be verifiable against the protocol by means such as model checking [9].

Security relates to both observability and controllability measures. The system must not allow unauthorized parties to have access to private medical information. Nevertheless, it is conceivable that a medical doctor can have access to all information in an emergency situation. At the same time, the 
system's operation should be fully protected by intrusions or interferences that can modify the system operation.

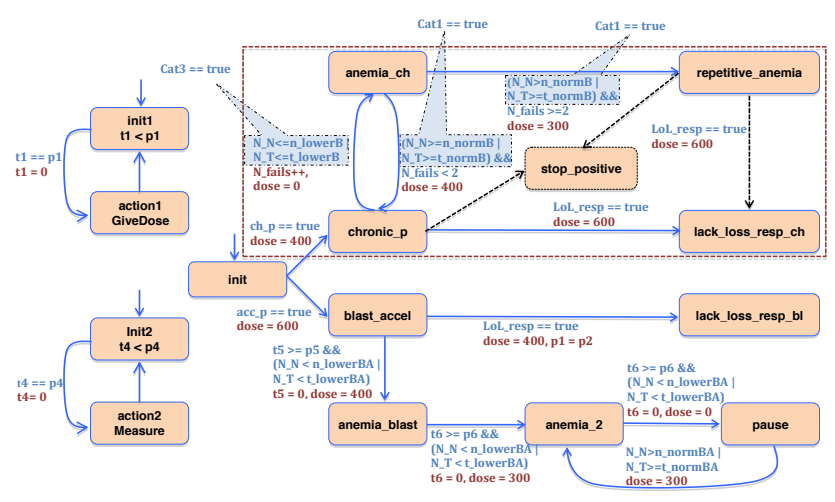

Figure 4: The dose adjustment formal protocol for Imatinib [11].

Dependability is a generic term that encompasses reliability and availability. It is important that the system has a very small and bounded probability of failure during its operation. The use of bio-sensors and biomaterials, that degrade with time and that interact with living cells and tissues, must be carefully analyzed to avoid unexpected failures. Moreover, it is expected that health management system have graceful degradation modes in case of local failures.

We expect the design of such systems to be effected by means of a rigorous design methodology, starting from formal high-level specifications and providing implementation models that are formally verifiable. Whereas this approach has been applied to electrical and electromechanical systems (e.g., aircrafts), the presence of biological materials and processes provides a new challenge in abstracting and modeling them.

\section{CONCLUSIONS}

E-health is going to revolutionize health care as much as information technology as changed our way of interacting. Thus it represents a broad area of research as well as a wide potential market for applications for wellbeing and health management. This review has just scratched the surface of a wide body of activities that includes both invasive and non-invasive sensing technologies, imaging, telemedicine, support for surgery as well as the crosspollination of these fields. The NanoTera.ch research initiative [10], funded by the Swiss government, is exploring some of these important themes. Similar research activities in the world are also contributing to giving a new meaning to the words 'electronic' and 'health' combined together, as enablers for a better society.

\section{ACKNOWLEDGEMENTS}

This work was supported in part by the ERC senior grant NanoSys ERC-2009-AdG-246810 and by the Swiss NanoTera.ch initiative.

\section{REFERENCES}

[1] C. Baj-Rossi et al. "Full Fabrication and Packaging of an Implantable Multi-panel Device for Monitoring of Metabolites in Small Animals', in IEEE Transactions on Biomedical Circuits and Systems, B, Vol. 8, Num. 6, pp. 891 - 898, 2014.

[2] S. Carrara, et al., "Do Carbon Nanotubes Contribute to Electrochemical Biosensing?,' in Electrochimica Acta, Vol. 128, pp. 102-112, 2014.

[3] A. Cavallini et al., "A Subcutaneous Biochip for Remote Monitoring of Human Metabolism: Packaging and Biocompatibility Assessment', IEEE Sensors Journal, Vol. 15, Num. 1, pp. 417-424, 2015.

[4] D. Y. Chiu et al., "A complete, hypermedia medical decision analysis support system," in Computer-Based Medical Systems, Proceedings of IEEE Seventh Symposium on, pp. 16-21, Jun 1994.

[5] E. Clarke and J. Wing, "Formal Methods: state of the art and Future Directions,', ACM Computing Surveys, Vol. 28, Num. 4, 1996.

[6] G. De Micheli, C. Boero and S. Carrara. "Implantable devices: the future of blood monitoring?", Editorial, in FSG Future Medicine Clinical Practice, Vol. 10, Num. 4, pp. 385-388, 2013.

[7] P. G. W. Keen and M. S. S. Morton, Decision Support Systems: An Organizational Perspective. AddisonWesley Pub. Co., 1978.

[8] G.Kong, D.-L.Xu, and J. B. Yang, "Clinical decision support systems: A review on knowledge representation and inference under uncertainties," International Journal of Computational Intelligence Systems, Vol. 1, Num. 2, pp. 159-167, 2008.

[9] K. McMillan, Symbolic Model Checking, Springer 1993.

[10] http://www.nano-tera.ch/

[11] A. Simalatsar, et al., "Representation of Medical Guidelines with a Computer Interpretable Model', in International Journal on Artificial Intelligence Tools (IJAIT), Vol. 23, Num. 3, 2014.

[12] A. Tobias and A. Jenkins, "Tutorial Review: Modern Biological Sensors', in R. Foerch et al., Surface Design: Applications in Bioscience and Nanotechnology, Wiley, 2009.

[13] F. Valgimigli, et al., "Evaluating the Clinical Accuracy of GlucoMen Day: A Novel Microdialysisbased Continuous Glucose Monitor," J. Diabetes Sci Technol., vol. 4, pp. 1182-1192, 2010.

[14] N. Widmer et al., "Population pharmacokinetics of Imatinib and the role of $\alpha 1$-acid glycoprotein," British Journal of Clinical Pharmacology, Vol. 62, Num. 1, pp. 97-112, 2006.

[15] W. You et al., "Personalized Drug Administrations Using Support Vector Machine", BioNanoScience, 2013. 